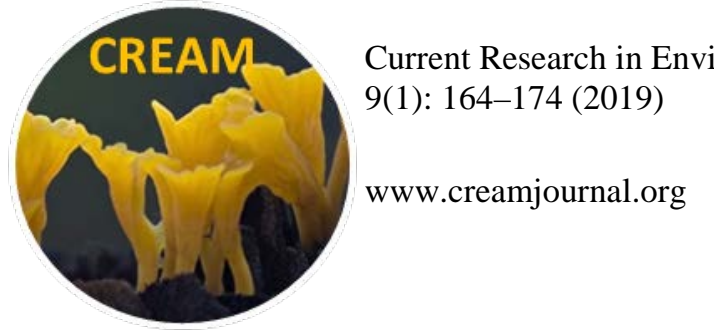

Current Research in Environmental \& Applied Mycology (Journal of Fungal Biology)

ISSN 2229-2225

\author{
Article \\ Doi 10.5943/cream/9/1/14
}

\title{
In vitro antagonistic activity of Argentinean isolates of Purpureocillium lilacinum on Nacobbus aberrans eggs
}

\author{
Gortari MC ${ }^{1,2 *}$, Hours RA ${ }^{2}$ \\ ${ }^{1}$ Buenos Aires Province Research Commission (CIC-PBA). Buenos Aires, Argentina. \\ ${ }^{2}$ Research and Development Center for Industrial Fermentations (CINDEFI), School of Science, La Plata National \\ University. La Plata, Argentina.
}

Gortari MC, Hours RA 2019 - In vitro antagonistic activity of Argentinean isolates of Purpureocillium lilacinum on Nacobbus aberrans eggs. Current Research in Environmental \& Applied Mycology (Journal of Fungal Biology) 9(1), 164-174, Doi 10.5943/cream/9/1/14

\begin{abstract}
In vitro interaction of three Argentinean isolates of Purpureocillium lilacinum (one isolated from a public park and two from agricultural soils) towards Nacobbus aberrans eggs was studied. After seven incubation days, the three isolates showed reproductive fungal structures fully developed whereas 80 to $100 \%$ of the eggs were infected. No significant differences among the three isolates were observed in fungal activity with respect to hatching and parasitism under the assayed test conditions ( $>0.05$ ). The hatching percentage in control plates increased during the evaluation period of the cultures, with no signs of fungal infection, showing significant differences as compared to the plates infected $(\mathrm{p}<0.05)$. Although the three isolates studied could be considered as potential agents for biological control of root-knot nematodes, it is necessary to further study some aspects in a wide range of experimental conditions in the laboratory, greenhouse and field so as to determine their real efficiency.
\end{abstract}

Key words - egg parasitism - nematophagous fungi - root-knot nematodes - tomato

\section{Introduction}

Total vegetable production in Argentina range from 8 to 10 million ton/year with more than 115 species cultivated. Most of these vegetables are destined to local and/or regional markets. In average, $65 \%$ of the production corresponds to 9 species, including tomato. The importance of tomato as a fresh or preserved food has given rise to the need for a comprehensive management of tomato crop and the implementation of the corresponding Good Agricultural Practices. Vegetable growers face several problems; one of them is the sanitary problem, which is usually managed from a reductionist point of view (crop-pest relationship), being the use of agro-chemicals the essential tool to control diseases and pests (Argerich \& Troilo 2011).

Phytonematodes constitute a limiting factor that has become more relevant in the last years, especially in crops under greenhouse conditions. Among them, cecidogenous (gall-formers) phytonematodes are the most important, not only due to their direct incidence on the crops but also due to their capacity to reproduce and persist in the environment. These nematodes are responsible for plant weakness and crop losses due to the presence of galls or "rosary-like beads" in the plant roots. The most important genera of cecidogenous phytonematodes are Meloidogyne and Nacobbus 
(Argerich \& Troilo 2011, Jones et al. 2013). Nacobbus aberrans (Thorne) Thorne and Allen or "the false root-knot nematode" is endemic in Argentina and other American countries where it produces great economic losses. This nematode affects a wide range of hosts, among which tomato crop, particularly when cultivated under greenhouse conditions, is one of the most attacked with losses that may range from 10 to $40 \%$ (Adlercreutz et al. 2008). Nacobbus aberrans presents a complex life-cycle, with migratory, vermiform juveniles and immature adults moving through the root and feeding on cells, causing cavities and lesions inside the root tissue. By contrast, the mature females are sedentary and induce partial dissolution of the cell walls and the fusion of cell protoplasts, resulting in specialized feeding sites called syncytia on which the "galls" are formed (Manzanilla López et al. 2002, Jones et al. 2013). Nacobbus aberrans is usually found in higher population densities than Meloidogyne sp. In addition, persistence of $N$. aberrans in the environment is related to the lack of commercial cultivars resistant to this nematode. Nacobbus aberrans is a quarantine pest (A1) subjected to multiple regulations in various parts of the world where its presence has not yet been reported (EPPO 1984).

The use of chemical pesticides, which are generally recognized as potentially toxic for humans and the environment, eventually constitutes a short-term solution to the problem of nematodes (Sabarwal et al. 2018). However, it finally results in the loss of soil biodiversity, the alteration of the trophic chains and the subsequent loss of the soil health (Kibblewhite et al. 2008). Substances used as chemical fumigants include Metam Sodium, Metam Potassium, Chloropicrin + 1,3-dichloropropene and methyl bromide whereas Aldicarb, Carbofuran, Etoprop and Fenamifos are currently employed as nematicides. On the other hand, Argentina has confirmed the Montreal Protocol, which states that methyl bromide (used mainly as soil fumigant) should be withdrawn from the market (Valeiro 2015).

In search of alternatives to this management practice, need arises of the new studies involving native microorganisms with antagonistic activity against the diverse stages of root-galling nematodes. The soil is a complex environmental system, where pathogenic and beneficial microorganisms influence plant growth and health. Using some of these microorganisms as biocontrol agents could result in a potential reduction in the use of agrochemical products and a contribution to a more sustainable use of the soil (Massart et al. 2015, van Lenteren et al. 2017).

Nematophagous fungi have attracted much attention among the potential biocontrol agents of phytonematodes. According to their action they can be classified in four groups (nematodetrapping, endoparasitic, egg and female-parasite, and toxin-producing fungi). Genera Verticillium, Paecilomyces, Pochonia, etc., are included in the group of egg- and female-parasitic fungi. Some of them are commercially produced and used in crop fields with highly variable and inconsistent performance. Pochonia chlamydosporia and Paecilomyces lilacinus have demonstrated to be the most effective (Dong \& Zhang 2006, Mubyana-John \& Wright 2011, Tranier et al. 2014).

Paecilomyces lilacinus (Thom) Samson, currently named Purpureocillium lilacinum (Thom) Luangsa-ard et al. (Luangsa-ard et al. 2011), is associated with antagonism towards sedentary stages of root-galling nematodes (eggs and females). It is an opportunistic parasite since its parasitic activity depends on the possibility of establishing contact with the "target" organism (Cannayane \& Sivakumar 2001, Bhat et al. 2009, Udo et al. 2014). Paecilomyces lilacinus 251 deposited in the Australian Government Analytical Laboratory (AGAL, N 89/030550) is recorded and commercialized as Bio-Act and is used as biocontrol agent for phytonematodes (von Erffa 2013). A great number of $P$. lilacinum isolates have been obtained from soil of different parts of the world, as well as from nematodes and insects, being this fungus one the most used in formulated nematicides (Dong \& Zhang 2006, Wilson \& Jackson 2013). The great diversity regarding environment and hosts suggests that with the same infection mechanism, the different isolates show differences regarding the efficiency of their antagonistic activity (Holland et al. 1999, Capparelli Cadioli et al. 2007).

The aim of the present study was to assess the in vitro antagonistic activity of three Argentinean isolates of $P$. lilacinum, obtained from soils with different characteristics, against $N$. aberrans eggs. 


\section{Materials \& Methods}

\section{Microorganisms and culture conditions}

Purpureocillium lilacinum LPSC \# 876, isolated from the soil of a public park (Paseo del Bosque, La Plata City, Buenos Aires Province, Argentina), was used. The fungus was isolated using an adapted soil sprinkling technique with eggs of Toxocara canis (Werner 1782) to bait fungal egg-parasites (Gortari et al. 2007). This isolate is deposited in the strain collection at the Spegazzini Institute Fungal Type Culture Collection (La Plata, Argentina).

Other two P. lilacinum isolates, designed Ls and Pv, were obtained from soil samples taken from an orchard (Pereyra Iraola Park, Buenos Aires Province, Argentina) using commercial chitin (coarse flakes) wrapped in a 50-mesh nylon cloth as a baiting bag (Tsay et al. 2006).

The three P. lilacinum isolates (LPSC \# 876, Ls and Pv) were cultured on potato dextrose agar (PDA) at $28 \pm 1{ }^{\circ} \mathrm{C}$ for 10 days. The conidia produced were extracted by adding $0.1 \%$ Tween 80 solution and the suspension obtained was diluted at a concentration of $\cong 1 \times 10^{6}$ conidia/mL before used.

\section{Taxonomic studies}

All fungal isolates were identified on the basis of their macro- and micro-morphological characteristics. Molecular confirmation was conducted by the Institute of Microbiology and Agricultural Zoology (IMYZA), National Institute of Agricultural Technology. Isolates were grown on potato dextrose broth (PDB) at $28 \pm 1{ }^{\circ} \mathrm{C}$ for 3 days. Total DNA was extracted using a DNeasy Plant Mini Kit (Qiagen, Germany) according to manufacturer's instructions. Amplification of the rDNA-ITS region (1 and 2 introns and 5.8S exon) using ITS1/ITS4 primers and of the TEF gene was carried out as described by White et al. (1990) and Luangsa-ard et al. (2011), respectively. The ITS and TEF dataset was combined and maximum likelihood analysis was performed using ClustalW in MEGA 5.1 (Tamura et al. 2011). In this context, Purpureocillium lilacinum (Luangsaard et al. 2011) was used as the taxonomic denomination for our study.

\section{Evaluation of fungal viability}

Viability of conidia was estimated from the germination rate (expressed as percentage) after a culture period ranging from 18 to $24 \mathrm{~h}$, at $28 \pm 1{ }^{\circ} \mathrm{C}$, in $1 \%$ water agar in a wet chamber. Aliquots of culture medium were placed on a sterile glass slide mounted on a V-shaped glass rod on a filter paper moistened with sterile distilled water inside a Petri plate $(9 \mathrm{~cm})$. Ten microliters of each conidial suspension $\left(\cong 1 \times 10^{6}\right.$ conidia/mL) were cultured on the solidified medium. After cultivation, conidia were observed under microscope $(400 \times)$ and the germination percentage was determined.

\section{Phytonematode}

Nacobbus aberrans was collected from soil samples from tomato crop fields cv. Elpida F1® (Syngenta AG, Switzerland) naturally parasitized in the Experimental Farm, School of Agriculture and Forestry, National University of La Plata. The taxonomic identification was conducted by the Laboratory of Plant Protection, Rama Caída Agricultural Experimental Farm (Mendoza-San Juan Regional Center, National Institute of Agricultural Technology) on the basis of morphological characteristics. The parasite was maintained and reproduced from a single mass of eggs (ME) on tomato plants cv. platense grown in pots under greenhouse conditions (Vovlas et al. 2007).

\section{Extraction of egg masses of $N$. aberrans}

After 55-60 days of cultivation, tomato plants were removed. Root pieces with galls were carefully washed with tap water and treated with a solution of Phloxine B (0.015 \%) for 20 min to stain the ME. The ME were then extracted under a stereoscopic microscope $(10 \times-50 \times$, Leica EZ5) with the help of a dissecting needle and placed in an Eppendorf test tube. The eggs were separated by dissolution of the gelatinous matrix of the $\mathrm{ME}$ with a $1 \mathrm{~mL}$ of $\mathrm{NaClO}(1 \%)$ solution by 
vortexing for $5 \mathrm{~min}$. Then, eggs were repeatedly washed with sterile distilled water and centrifuged $(14,000 \times$ g, $1 \mathrm{~min})$ to completely remove residual $\mathrm{NaClO}$. The number of eggs/mL was calculated by counting one aliquot $(10 \mu \mathrm{L})$ using a stereoscopic microscope.

\section{In vitro antagonistic tests}

Each isolate of $P$. lilacinum was in vitro assessed for their antagonism towards $N$. aberrans eggs employing the direct dual confrontation method using the following two culture techniques simultaneously: 1) in plates and 2) on slides:

1) In plates $(\varnothing=5 \mathrm{~cm})$ containing water agar $(1 \%)$ supplemented with chloramphenicol (1 $\mathrm{g} / \mathrm{L})$. Equal amounts $(10 \mu \mathrm{L})$ of the suspension of $N$. aberrans eggs and of the conidial suspension of each of the fungal isolates were spread onto the agar. Plates containing $N$. aberrans eggs or with each isolate were used as controls. Each treatment was conducted in triplicate. Plates were incubated at $28 \pm 1{ }^{\circ} \mathrm{C}$ and observed daily using a stereoscopic microscope for a week.

The variables analyzed were: hatching percentage $=100 \times$ juveniles / (juveniles + eggs), and infected eggs percentage $=100 \times$ infected eggs / (total of eggs). Infected eggs were considered as the proportion of eggs which were totally invaded by fungal hyphae with the embryonic or larval content disintegrated.

2) On slide cultures: three segments of $1 \%$ water agar ( 1 for each isolate) of approximately 2 $\mathrm{cm} \times 2 \mathrm{~cm}$ on a side were placed onto a sterile glass slide, inoculated as mentioned for the above technique and cultured in wet chambers (Mubyana-John \& Wright 2011). Each glass slide was placed on a V-shaped glass rod as a stand in a Petri plate $(\varnothing=90 \mathrm{~mm})$ containing a filter paper moistened with sterile distilled water. Each block was used for the microscopic observation and for capture of photographic images after stopping the infection process by adding some drops of Amman lactophenol blue solution (1\%)

\section{Statistical analysis}

Antagonistic tests using the three fungal isolates simultaneously were repeated three times and data shown are average of triplicates \pm SD. Statistical analysis of the tests was performed using the Infostat statistical software (Di Rienzo et al. 2008). Data (in percentage) were subjected to arcsine transformation and analyzed according to standard procedure for analysis of variance (ANOVA) and Tukey’s multiple comparison tests.

\section{Results}

After $18 \mathrm{~h}$ of cultivation, conidia germination for the three isolates of $P$. lilacinum ranged from 87 to $100 \%$ whereas after $24 \mathrm{~h}$, it accounted for $100 \%$. It should be noted that the suspension of $N$. aberrans eggs used as inoculum contained eggs at different stages of embryonic development (Figs 1, 2). Also, some "empty" eggs were observed, probably due to previous hatching and/or anomalies in their development. The initial hatching percentage ranged from 3.3 to $7.9 \%$.

In vitro tests revealed that the three $P$. lilacinum isolates show antagonism against $N$. aberrans eggs, regardless their embryonic stage. The morphological changes microscopically visualized $(100 \times$ and $400 \times)$ varied with time course of the interaction experiments and were similar for the three fungal isolates evaluated. However, these changes were not uniform due to the characteristics of the egg suspension used. Eggs surrounded by hyphae were observed after $24 \mathrm{~h}$ (Fig. 3a). After $48 \mathrm{~h}$, the eggs showed signs of infection (Fig. 3b) whereas conidiophore development and conidia production were detected between 48-72 h of culture. A high proportion of eggs (75 to $86 \%$ ) showed variable signs of infection (Fig. 3c-d) while larval stages remained normal (not seen in the photographs).

After 5 days, reproductive fungal structures were fully developed (Fig. 3e-f) whereas 80 to $100 \%$ of the eggs were infected. It was observed that the infected eggs were highly invaded by hyphae, vacuolated with deformed edges and with no observable remains of the embryo and/or larva. Hatching remained within the initial levels and infected larvae were exceptionally detected. No significant differences among the three isolates were observed in fungal activity with respect to 
hatching $(\mathrm{p}=0.83)$ and parasitism $(\mathrm{p}=0.36)$ under these test conditions. The hatching percentage in control plates increased during the evaluation period of the cultures, with no signs of fungal infection, showing significant differences as compared to the plates infected with the fungus $(\mathrm{p}=$ $<0.0001$ ) (Table 1).

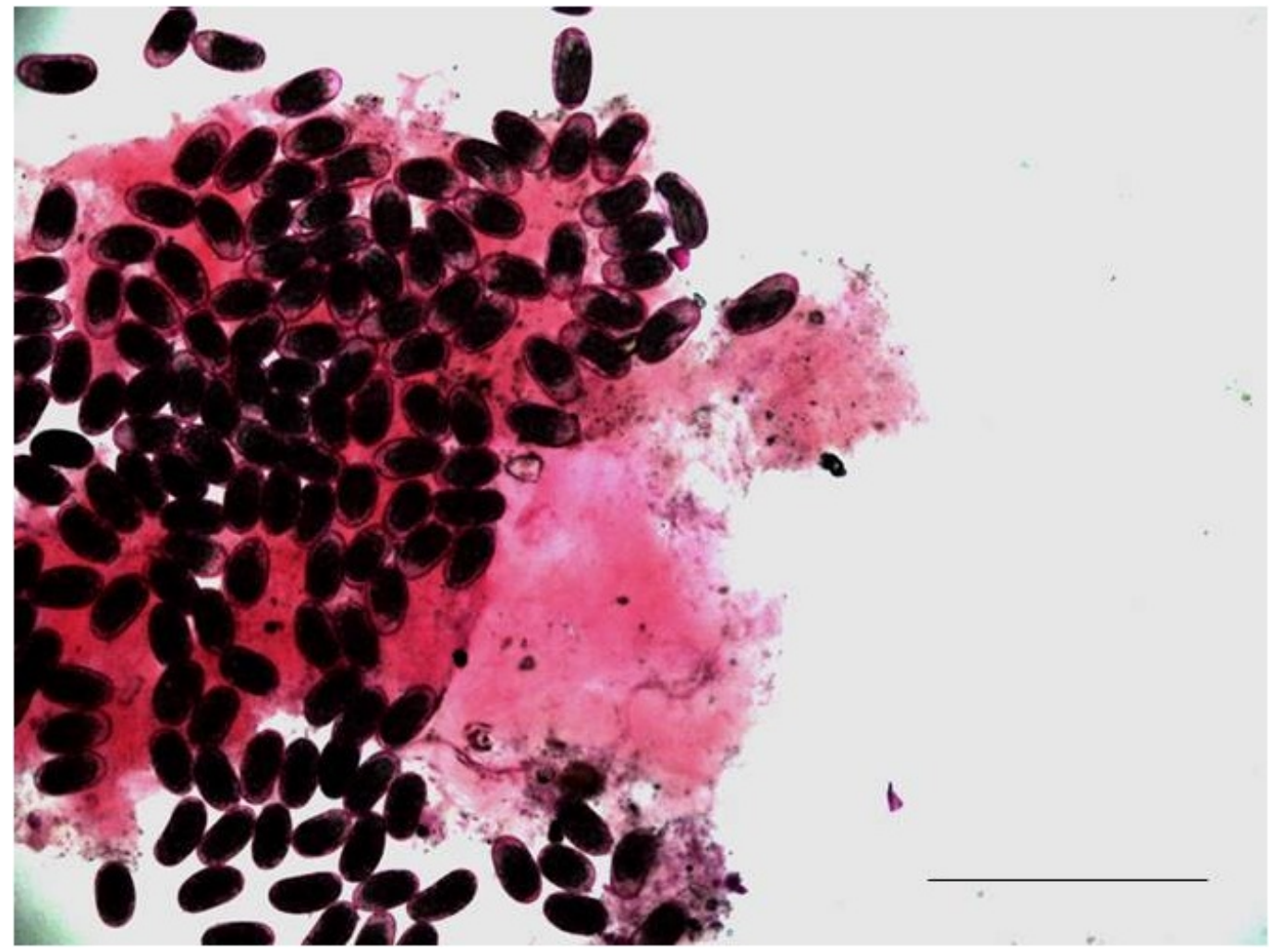

Fig. 1 - An egg mass of Nacobbus aberrans stained with Phloxine B. Scale bar: $200 \mu \mathrm{m}$.

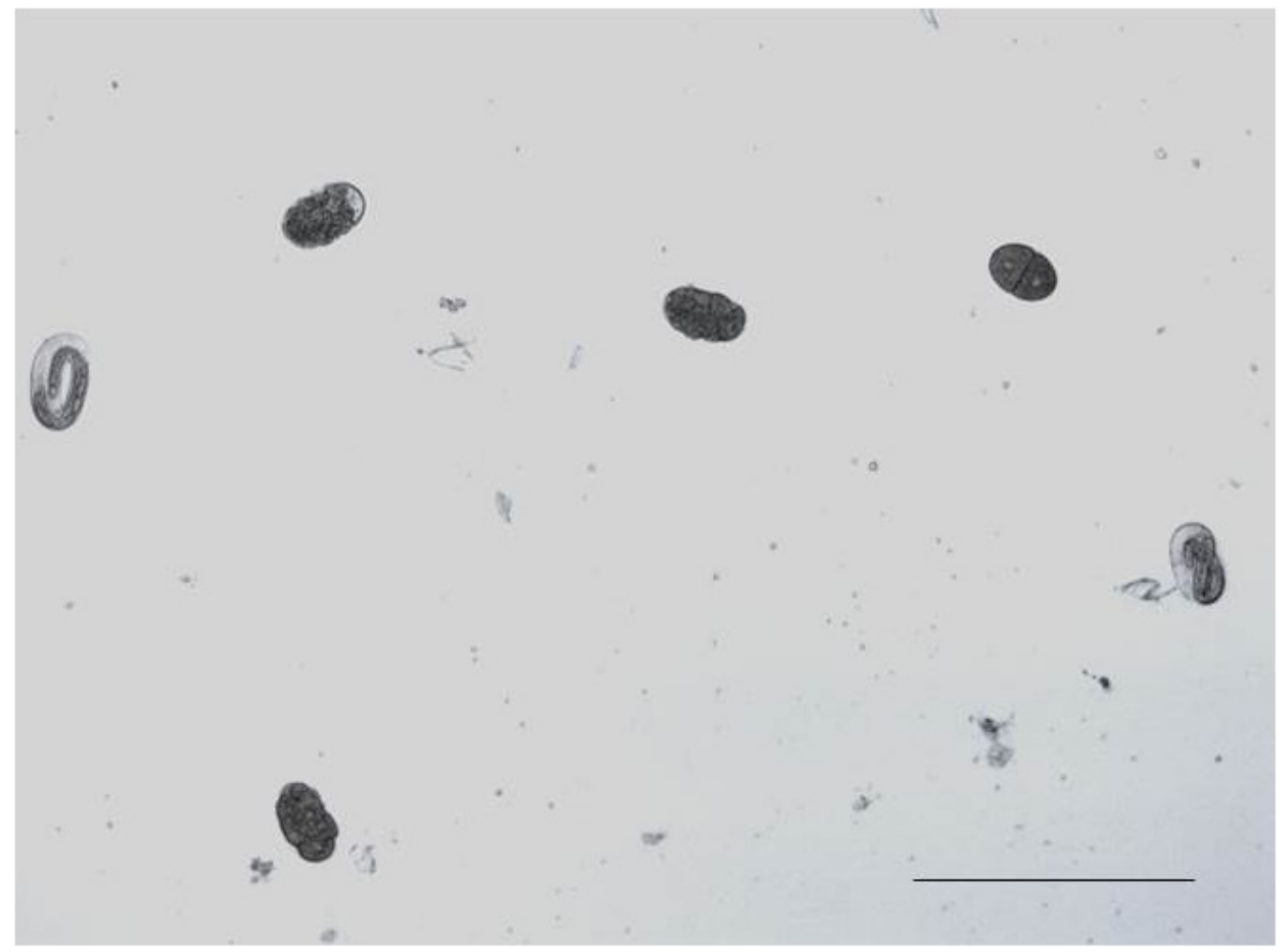

Fig. 2 - Nacobbus aberrans eggs used as inoculum. Scale bar: $200 \mu \mathrm{m}$. 

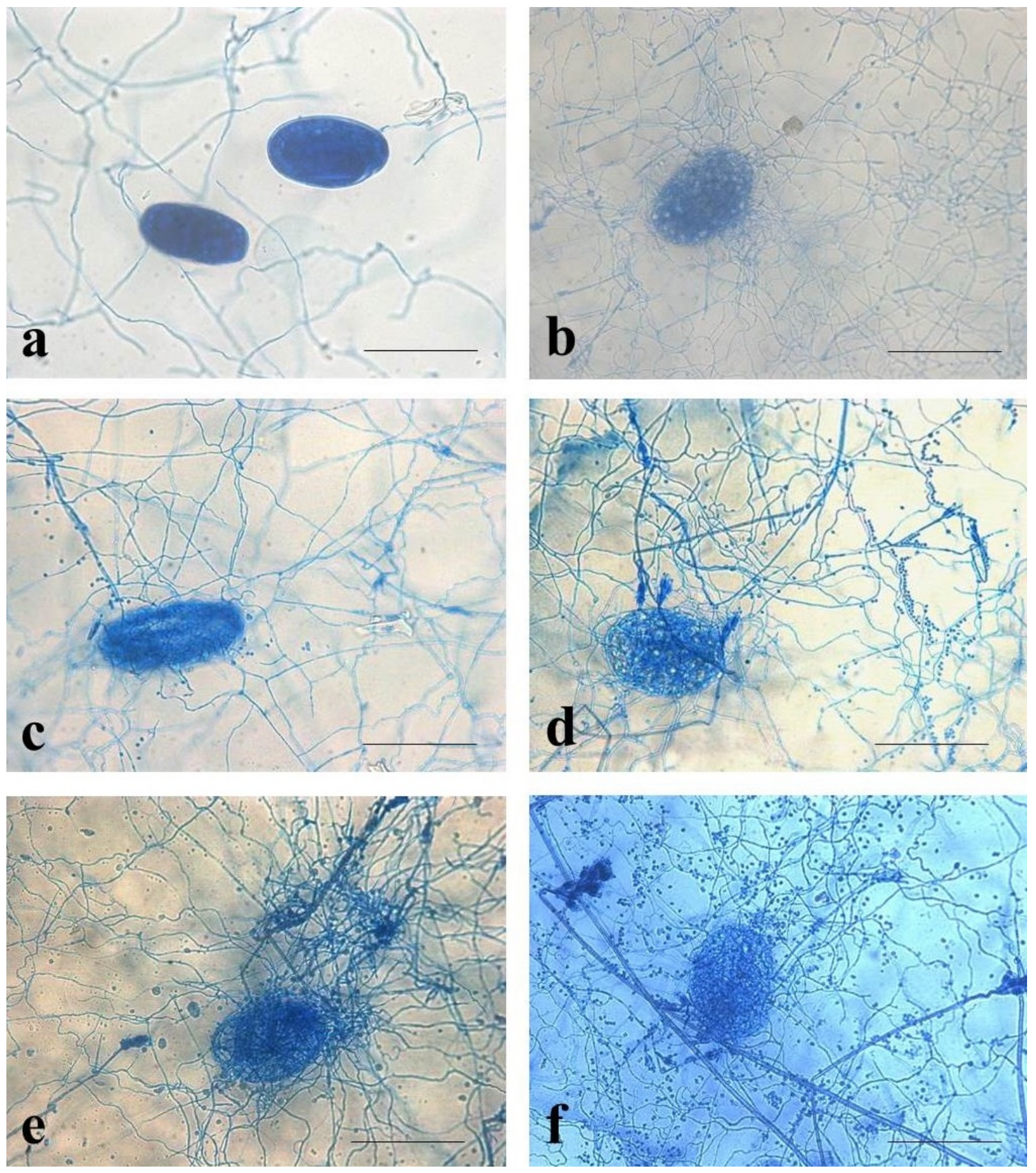

Fig. 3 - Nacobbus aberrans eggs infected by Purpureocillium lilacinum LPSC \# 876 stained with lactophenol blue after different incubation times: a 24 h. b 48 h. c 72 h. d 96 h. e 120 h. f 144 h. Scale bar: $50 \mu \mathrm{m}$.

Table 1 In vitro antagonism of Purpureocillium lilacinum isolates to Nacobbus aberrans eggs.

\begin{tabular}{|l|l|l|l|l|l|l|l|}
\hline \multirow{2}{*}{ Isolate } & \multirow{2}{*}{ Test } & \multicolumn{3}{|c|}{ Eggs parasitized (\%) } & \multicolumn{3}{c|}{ Egg hatch rate (\%) } \\
\cline { 3 - 8 } & & Average & Minimum & Maximum & Average & Minimum & Maximum \\
\hline P. lilacimun LPSC \# 876 & 1 & $87 \pm 4^{\mathrm{a}}$ & 84 & 91 & $6 \pm 4^{\mathrm{a}}$ & 2 & 10 \\
\hline & 2 & $92 \pm 6^{\mathrm{a}}$ & 87 & 98 & $5 \pm 4^{\mathrm{a}}$ & 0 & 7 \\
\hline & 3 & $96 \pm 4^{\mathrm{a}}$ & 93 & 100 & 0 & 0 & 0 \\
\hline P. lilacimun Ls & 1 & $84 \pm 3^{\mathrm{a}}$ & 80 & 85 & $9 \pm 4^{\mathrm{a}}$ & 7 & 14 \\
\hline & 2 & $87 \pm 2^{\mathrm{a}}$ & 84 & 89 & $9 \pm 2^{\mathrm{a}}$ & 7 & 11 \\
\hline & 3 & $91 \pm 5^{\mathrm{a}}$ & 86 & 97 & $1^{\mathrm{a}}$ & 0 & 1 \\
\hline
\end{tabular}


Table 1 Continued.

\begin{tabular}{|l|l|l|l|l|l|l|l|}
\hline \multirow{2}{*}{ Isolate } & \multirow{2}{*}{ Test } & \multicolumn{3}{c|}{ Eggs parasitized (\%) } & \multicolumn{3}{c|}{ Egg hatch rate (\%) } \\
\cline { 3 - 8 } & & Average & Minimum & Maximum & Average & Minimum & Maximum \\
\hline P. lilacimun Pv & 1 & $83 \pm 1^{\mathrm{a}}$ & 82 & 84 & $6 \pm 1^{\mathrm{a}}$ & 5 & 7 \\
\hline & 2 & $90 \pm 9^{\mathrm{a}}$ & 85 & 100 & $5 \pm 2^{\mathrm{a}}$ & 2 & 7 \\
\hline Control & 3 & $85 \pm 4^{\mathrm{a}}$ & 80 & 88 & $1^{\mathrm{a}}$ & 0 & 2 \\
\hline & 1 & - & - & - & $73 \pm 2^{\mathrm{b}}$ & 71 & 75 \\
\hline & 2 & - & - & - & $66 \pm 3^{\mathrm{b}}$ & 63 & 69 \\
\hline & 3 & - & - & - & $65 \pm 8^{\mathrm{b}}$ & 60 & 75 \\
\hline
\end{tabular}

Values in the same column followed by the same letter are not significantly different.

\section{Discussion}

Nacobbus aberrans is a phytonematode geographically restricted. Its life cycle begins with eggs laid in a gelatinous matrix (up to $\cong 800$ per ME) and is completed after 30-100 days depending mainly on temperature. Nematodes' eggs can persist in the soil for long periods of time and hatch in a response to environmental stimuli, such as temperature, moisture, etc. (Manzanilla López et al. 2002). The egg-parasitic fungi are the most promising candidates as biocontrol agents of phytonematodes due to the fact that their most important biological activity is exerted on one of the most resistant stages of the nematodes' life cycle; in addition, they are also the most efficient in reducing nematode populations (Capparelli Cadioli et al. 2007).

Since the first report on an ovicidal fungus (Kühn 1877), there have been a large number of studies and evaluations of $P$. lilacinum as potential biological control agent of different species and genera of nematodes (Siddiqui \& Mahmood 1996). The three fungal isolates evaluated here were obtained from environments associated with the natural presence of eggs of diverse soil nematodes. $P$. lilacinum LPSC \# 876 was isolated from the soil of a public place contaminated with $T$. canis eggs, a nematode of dogs, which usually results on a zoonotic infection (toxocariosis). On the contrary, $P$. lilacinum Ls and $\mathrm{Pv}$ were isolated from an agricultural soil where plant parasitic nematodes predominate. In the present work, we attempted to evaluate if this substantial difference in the origin of the isolates could affect their antagonistic effect onto $N$. aberrans eggs considering their potential as biocontrol agents.

As far as the authors are aware, the available literature does not include in vitro interaction studies between $P$. lilacinum and $N$. aberrans eggs. Therefore, for the discussion we have chosen those already published results we considered as the most similar in relation to experimental conditions, organisms used, etc.

Conidial viability is one of the quality parameters used to evaluate the potential of a fungal preparation as a biological control agent. A fungal biopesticide of good quality should have a high concentration of viable conidia. In our case, conidia from the tested isolates germinated within 18 to $24 \mathrm{~h}$ in good accordance with Núñez-Camargo et al. (2012).

In our study, antagonistic activity towards the $N$. aberrans eggs was observed in the three isolates analyzed. In general, the antagonistic activity (determined by the number of infected eggs) increases with time course of the experiment in well accordance with the results of others authors (Holland et al. 1999, Vergara Alzate et al. 2012). However, it should be taken into account that an extremely prolonged experimental time may alter the interpretation of the results. Some changes in the organisms studied may depend on the experimental conditions: duration of the test, high temperatures, loss of substrate moisture, absence of the host organism, presence of toxic compounds in the environment, etc. (Moosavi et al. 2010, Vergara Alzate et al. 2012).

The ME of $N$. aberrans contains eggs in all stages of embryonic development indicating that the oviposition period is prolonged. Our results showed that under the laboratory conditions used, $P$. lilacinum acts against all stages of embryonic development (including those containing formed juveniles) in agreement with most of the experimental references considered (Holland et al. 1999, Dong \& Zhang 2006, Núñez-Camargo et al. 2012). Pau et al. (2012) also observed that the eggs were more sensitive during the initial developmental stages. However, in our case, this is difficult 
to confirm since we do not know the exact period of embryonic development in which the eggs are besides hatching is highly dependent on temperature. The activity against any of the embryonic stages is regarded as a good characteristic for a potential biocontrol agent since its action would be prolonged in time along with the differential development of the eggs (Holland et al. 1999).

Although the experimental conditions are comparable but not the same, different authors conclude that $P$. lilacinum is a fungus with an important ovicidal activity and that under certain circumstances it can also parasitize other stages of the nematode's life cycle (Holland et al. 1999, Sun et al. 2006, Bhat et al. 2009, Núñez-Camargo et al. 2012, Pau et al. 2012, Vergara Alzate et al. 2012). Holland et al. (1999) proposed that the fungal infection of the larval stages may not be significant since a long period of their life occurs inside the plant tissues, where they are protected from the fungal activity. However, some juvenile stages of $N$. aberrans act as resistant stages (and may persist a long time in the environment). On the other hand, some researchers have demonstrated that $P$. lilacinum behaves as an epiphyte and as an endophyte (Bhat et al. 2009). In this context, the study of the behavior of $P$. lilacinum onto juvenile stages of $N$. aberrans should be further studied.

Purpureocillium lilacinum LPSC \# 876, Ls and Pv demonstrated high and significant parasitism on $N$. aberrans eggs as compared to control but no significant among themselves. For the three isolates evaluated the percentage of egg-infection was close to $80-100 \%$ in good agreement with other reports. In a research study conducted in Spain the in vitro activity of phytonematode egg-parasitic fungi was compared under different conditions. Paecilomyces lilacinus showed the highest pathogenicity (100\% of infected eggs) and the highest severity (ca. 40 penetrating hyphae/egg) (Olivares-Bernabeu \& López-LLorca 2002). Sun et al. (2006) evaluated the antagonistic potential of different fungi associated with Meloidogyne spp. eggs. The most frequently isolated fungus was $P$. lilacinus with $100 \%$ of parasitism towards eggs in the pathogenesis tests. Capparelli Cadioli et al. (2007) analyzed the parasitism of 31 isolates of $P$. lilacinus towards $M$. paranaensis eggs and reported that the percentages of parasitism ranged from 56.0 to $98.7 \%$. Moosavi et al. (2010) conducted in vitro studies using strains of other ovicidal fungi, Pochonia spp., against M. javanica eggs. The percentage of pathogenicity ranged from 39 to $95 \%$ of infected eggs. The range observed in the percentage of infected eggs indicated differences in the pathogenicity of the strains. Pau et al. (2012) studied the antagonistic activity of the native isolates of $P$. lilacinus against different stages of $M$. incognita. The parasitism against the eggs was significant: 78.5, 73.4 and 66 \% for Paecilomyces PLA, PLM and PLB, respectively, and no differences among them were observed. Vergara et al. (2012) studied the in vitro effect of $P$. lilacinum Pl-11 and Pochonia chlamydosporia var. catenulata, both in their commercial form, on Radopholus similis. The infection degree of the eggs increased from $24 \mathrm{~h}$ to $120 \mathrm{~h}$ (final evaluation) reaching 79 and $85 \%$ of infection for P. lilacinum and P. chlamydosporia, respectively. NúñezCamargo et al. (2012) isolated local fungi associated with Globodera rostochiensis and evaluated the pathogenicity of $P$. lilacinum towards eggs and juveniles of the parasite. The authors studied the infection process for 5 days and reported $100 \%$ mortality of infected eggs and juveniles J1 after this period.

In this study, egg hatch was not significantly different among the three isolates evaluated but significant with the control (65-73\%). This result may be considered analogous to some above mentioned investigations (Sun et al. 2006, Pau et al. 2012). Khan et al. (2004) treated M. javanica eggs with protease, chitinase (enzymatic complex) and a mixture of both enzymes, all of them partially purified from a liquid culture of $P$. lilacinus 251. These authors observed that the enzymatic activity of the fungus (protease and chitinase enzymes, either individually or in combination) negatively affects embryogenesis, at any stage, preventing the $\mathrm{J} 2$ hatching process.

Some authors reported that the characteristics of the soil where the fungi were isolated might determine variations in the antagonistic activity (Olivares-Bernabeu \& López LLorca, 2002, Capparelli Cadioli et al. 2007, Moosavi et al. 2010). However, in our investigation, non-significant differences were observed among the P. lilacinum isolates analyzed (LPSC \# 876, Ls and Pv) obtained from soils with very different characteristics although from the same geographic region 
(similar edaphic and climatic conditions). It should be noted that the P. lilacinum Ls and Pv isolates were obtained from samples of agricultural soils with natural infection with $N$. aberrans. In natural environments, a fungus could show a higher degree of antagonism when it is adapted to the local microflora of the phytoparasites and therefore it could reach a greater development, contributing to the natural control of phytoparasites (Moosavi et al. 2010).

The fungal infection of phytonematode eggs occurs by means of mechanical pressure as well as enzymatic digestion (Holland et al. 1999, Khan et al. 2004, Moosavi et al. 2010). Taking into account the infection process of the eggs for the fungal isolates used and that the three isolates evaluated are protease and chitinase producers (Gortari et al. 2014), it could be stated that they present the same action mechanism suggested for the fungal strains used in the aforementioned studies.

The three isolates studied could be considered as potential candidates for biological control of root-knot nematodes. Nevertheless, it is necessary to further study some aspects in a wide range of experimental conditions in the laboratory, greenhouse and field so as to determine their real efficiency. However, in vitro studies may be a first step in the understanding of the events that are part of the biological activity of antagonistic fungi. Success or failure of the biological control agent application depends on several factors and their effect will be associated with a complex interaction among the host plant - target nematode - soil - biological control agent - environment. In this sense, the studies on P. lilacinum - N. aberrans - tomato interaction constitute an important step for the understanding of the interaction and the evaluation of different management strategies that reduce the environmental impact of the agrochemicals.

\section{Acknowledgements}

The authors thank Guillermo Cap and Andrés Nico for providing the soil and N. aberrans samples, respectively, used in this study. This investigation was supported by National University of La Plata (UNLP, Grant 11/X650 to R. Hours).

\section{References}

Adlercreutz EGA, Chaves E, Mondino E, Szczesny A. 2007 - Fluctuacion poblacional de juveniles del segundo estadio de Nacobbus aberrans y Meloidogyne sp. bajo condiciones de invernaculos (Período sept. 2004/oct. 2007). Congreso Argentino de Horticultura 31. Mar del Plata, Buenos Aires, Argentina.

Argerich C, Troilo L. 2011 - Manejo del cultivo para cualquier sistema de producción de tomate. En: Argerich C, Troilo L, editores. Manual de buenas prácticas agrícolas en la cadena de tomate. Buenos Aires: FAO.

Bhat MY, Muddin H, Bhat NA. 2009 - Histological interactions of Paecilomyces lilacinus and Meloidogyne incognita on bitter gourd. The Journal of American Science 5(1), 8-12.

Cannayane I, Sivakumar CV. 2001 - Nematode egg-parasitic fungus I: Paecilomyces lilacinus-A review. Agricultural Reviews 22(2), 79-86.

Capparelli Cadioli M, Santiago DC, Thibes Hoshino A, Homechin M. 2007 - Crescimento micelial e parasitismo de Paecilomyces lilacinus sobre ovos de Meloidogyne paranaensis em diferentes temperaturas “in vitro”. Ciência e Agrotecnologia 31(2), 305-311.

Di Rienzo JA, Casanoves F, Balzarini MG, González L et al. 2008 - Infostat. Programa de análisis estadísticos. Grupo Infostat, Facultad de Ciencias Agropecuarias, Universidad Nacional de Córdoba, Argentina.

EPPO. 1984 - Data sheets on quarantine organisms. No. 144, Nacobbus aberrans. EPPO Bull 14, 61-66.

Dong LQ, Zhang KQ. 2006 - Microbial control of plant-parasitic nematodes: a five-party interaction. Plant Soil 288, 31-45.

Gortari MC, Cazau C, Hours R. 2007 - Hongos nematofagos de Toxocara canis, en un paseo público de La Plata, Argentina. Revista Iberoamericana de Micología. 24, 24-28. 
Gortari MC, Galarza B, Hours R. 2014 - Actividad quitinasa de Purpureocillium lilacinum en medio sólido. Lilloa 51, 182.

Holland RJ, Williams KL, Khan A. 1999 - Infection of Meloidogyne javanica by Paecilomyces lilacinus. Nematology 1(2), 131-139.

Jones JT, Haegeman A, Danchin EGJ, Gaur HS et al. 2013 - Top 10 plant-parasitic nematodes in molecular plant pathology. Molecular Plant Pathology doi: 101111/mpp.12057

Khan A, Williams KL, Nevalainen HKM. 2004 - Effects of Paecilomyces lilacinus protease and chitinase on the eggshell structures and hatching of Meloidogyne javanica juveniles. Biological Control 31, 346-352.

Kühn J. 1877 - Vorlaufiger Bericht uber die bisherigen Ergebnisse der seit dem jahre 1875 in Aftrage des Vereins fur Ruberzucher Industrie aus gegiihrten versuche zue Ermittelung der ursacho der Rubenmudiqueit des Boden und Zur Er Forschung der Nature Nematoden. Z Ver Ruben Ind Dent Reich (Ohne Band) 452-457. Fide Siddiqui and Mahmood (1996).

Kibblewhite MG, Ritz K, Swift MJ. 2008 - Soil Health in agricultural systems. Philosophical transactions of the Royal Society B 363, 655-701.

Luangsa-ard J, Houbraken J, Van Doorn T, Hong SB et al. 2011 - Purpureocillium, a new genus for the medically important Paecilomyces lilacinus. FEMS Microbiology Letters 321, 141149.

Manzanillla López RH, Costilla MA, Doucet M, Franco J et al. 2002 - The genus Nacobbus Thorne \& Allen, 1944 (Nematoda: Pratylenchidae): Systematics, distribution, biology and management. Nematropica 32(2), 149-227.

Massart S, Martinez-Medina M, Jijakli MH. 2015 - Biological control in the microbiome era: Challenges and opportunities. Biological Control 89, 98-108.

Moosavi MR, Zare R, Zamanizadeh HR, Fatemy S. 2010 - Pathogenicity of Pochonia species on eggs of Meloidogyne javanica. Journal of Invertebrate Pathology 104, 125-133.

Mubyana-John T, Wright MG. 2011 - Fungi and their use in the possible control of nematodes in Botswana soils. African Journal of Plant Science and Biotechnology 5(1), 33-40.

Núñez-Camargo MC, Carrión G, Núñez-Sánchez AE, López-Lima JD. 2012 - Evaluación de la patogenicidad in vitro de Purpureocillium lilacinum sobre Globodera rostochiensis. Tropical and Subtropical Agroecosystem 15(2), 126-134.

Olivares-Bernabeu CM, López-Llorca LV. 2002 - Fungal egg-parasites of plant-parasitic nematodes from Spanish soils. Revista Iberoamericana de Micología 19, 104-110.

Pau CG, Leong CTS, Wong L, Eng M et al. 2012 - Isolation of indigenous strains of Paecilomyces lilacinus with antagonistic activity against Meloidogyne incognita. International Journal of Agriculture and Biology 14, 197-203.

Sabarwal A, Kumar K, Singh RP. 2018 - Hazardous effects of chemical pesticides on human healtCancer and other associated disorders. Environmental Toxicology and Pharmacology 63,103114.

Siddiqui IA, Mahmood I. 1996 - Biological control of plant parasitic nematodes by fungi: a review. Bioresource Technology 58, 229-239.

Sun MH, Gao L, Shi YS, Li BJ, Liu XZ. 2006 - Fungi and actinomycetes associated with Meloidogyne spp. eggs and females in China and their biocontrol potential. Journal of Invertebrate Pathology 93, 22-28.

Tamura K, Peterson D, Peterson N, Stecher G et al. 2011 - MEGA5: Molecular evolutionary genetics analysis using maximum likelihood, evolutionary distance, and maximum parsimony method. Molecular Biology and Evolution 28, 2731-2739.

Tranier MS, Pognant-Gros J, De la Cruz Quiroz R, Aguilar Gonzalez NA et al. 2014 - Commercial biological control agents targeted against plant-parasitic root-knot nematodes. Brazilian Archives of Biology and Technology 57(6), 831-841.

Tsay TT, Chen P, Wu WS. 2006 - A new method for isolating and selecting agents with high antagonistic ability against plant parasitic nematodes. Plant Pathology Bulletin 15, 9-16. 
Udo IA, Osai EO, Ukeh DA. 2014 - Management of root-knot disease on tomato with bioformulated Paecilomyces lilacinus and leaf extract of Lantana camara. Brazilian Archives of Biology and Technology 57(4), 486-492.

Valeiro A. 2015 - CIRAD, Una Plataforma de Cooperación Internacional en I\&D. Revista Industrial y Agrícola de Tucumán 92 (1), 62-68.

van Lenteren JC, Bolckmans K, Köhl J, Ravensberg WJ, Urbaneja A. 2017 - Biological control using invertebrates and microorganisms: plenty of new opportunities. BioControl DOI 10.1007/s10526-017-9801-4

Vergara Alzate D, Guzmán Piedrahita OA, Leguizamón Caycedo J. 2012 - Efecto in vitro de Purpureocillium lilacinum (Thom) Luangsa-Ard et al. y Pochonia chlamydosporia (Goddard) Zare y Gams sobre el nematodo barrenador Radopholus similis (Cobb) Thorne. Agronomía 20(2), 25-36.

von Erffa M. 2013 - Effective and selective control of plant parasitic nematodes with Paecilomyces lilacinus 251. Bayer CropScience 1-15.

Vovlas N, Nico AI, De Luca F, De Giorgi C, Castillo P. 2007 - Diagnosis and molecular variability of an Argentinean population of Nacobbus aberrans with some observations on histopathology in tomato. Journal of Nematology 39(1), 17-26.

White TJ, Bruns T, Lee S, Taylor J. 1990 - Amplification and direct sequencing of fungal ribosomal RNA genes for phylogenetics. In: Innis MA, Gelfand DH, Sninsky JJ, White TJ (editors). PCR protocols: a guide to methods and applications. San Diego: Academic. p. 315322.

Wilson MJ, Jackson TA. 2013 - Progress in the commercialization of bionematicides. Biocontrol 58, 715-722. 